Conclusion Patients with MC are diagnosed within a median of just over 3 months of symptom onset. Some patients are still referred and diagnosed long after symptom onset. There may be patients with chronic diarrhoea in the community who are undiagnosed. There is some variation in prescribing practice for MC. Standardisation of management pathway in the UK would assist with management (Münch A, et al. Frontline Gastroenterology 2020;11:228-234).

\section{PTH-51 PATIENT SAFETY MATTERS: CARE CLOSER TO HOME IN GASTROENTEROLOGY HIGH COST DRUG MANAGEMENT DURING COVID-19}

${ }^{1}$ Sapna Gohil*, ${ }^{1}$ Arti Soni, ${ }^{1}$ Maimuna Ahmed, ${ }^{1}$ Nikki Lovell, ${ }^{2}$ Duncan Loft, ${ }^{2}$ Esther Unitt, ${ }^{1}$ Hardeep Bagga. 'Pharmacy, University Hospitals Coventry and Warwickshire NHS Trust, Coventry, UK; ${ }^{2}$ Gastroenterology, University Hospitals Coventry and Warwickshire NHS Trust, Coventry, UK

\subsection{6/gutjnl-2021-BSG.366}

Introduction The Covid-19 pandemic has been a strong catalyst in driving the reconfiguration of gastroenterology outpatient models of High Cost Drug (HCD) delivery. This service evaluation aims to explore the impact of multiple quality improvement initiatives synchronously implemented by the HCD pharmacists and the gastroenterology clinical team, to improve medication safety and HCD treatment access.

Methods The Plan-Do-Study-Act (PDSA) quality improvement methodology was applied to three key service areas, from March 2020 to May 2021: i) 95 Hepatitis B (HB) patients receiving FP10 HCD prescriptions were reviewed by the clinical team for switch to the hospital outpatient pharmacy model (HOP), enabling HCD collection from their local pharmacy. Conversely, Hepatitis $\mathrm{C}(\mathrm{HCV})$ patients struggled to reach the HOP. To improve treatment access, 29 eligible HCV patients were offered HCV HCD homecare (HC) delivery, enabling direct delivery to the patient's preferred address ii) development of a digital pharmacy database to record queries and pharmacist interventions supporting safety monitoring for 450 HC gastroenterology patients iii) exploration of alternative HCD formulations available via HC.

Results $100 \%$ of the eligible $\mathrm{HCV}$ patients agreed to the HC model and $100 \%$ of $\mathrm{HB}$ patients agreed to the HOP. HCD pharmacists helped to resolve $114 \mathrm{HC}$ prescription queries in regard to incomplete blueteq proformas, delayed clinic followup reviews and blood monitoring. Subcutaneous (SC) formulations of vedolizumab and infliximab recently became available for self-administration (SA) via $\mathrm{HC}$, as options for eligible inflammatory bowel disease (IBD) patients considered to be at-risk of Covid-19. 41 patients receiving intravenous (IV) vedolizumab at the hospital were eligible for switch to SC via HC. 26/41 patients agreed to switch. 2/26 patients switched back to IV as 1 patient $=$ reported IBD flare and 1 patient $=$ injection site reaction. $15 / 41$ patients refused the switch. IV infliximab patients eligible for switch to SC are currently being reviewed. 72 new starter IBD patients were identified for potential commencement of the new cost-effective adalimumab biosimilar via HC.

Conclusions Gastroenterology HCD prescriptions are now being processed safely via two main outpatient delivery models, both governed by robust contractual arrangements. Improved clinical monitoring has helped to reduce the risk of adverse events, thereby improving medication safety. These simple initiatives also delivered prescribing cost efficiencies in excess of $£ 400,000$ ensuring best use of NHS resources.

\section{PTH-52 PBC MANAGEMENT IN A RURAL SETTING. HOW DO WE COMPARE TO NATIONAL STANDARDS?}

Stewart McDonald* ${ }^{*}$ Eugene Campbell. Western Health and Social Care Trust, Enniskillen, UK

\subsection{6/gutjnl-2021-BSG.367}

Introduction The South West Acute Hospital (SWAH) serves the most rural population in Northern Ireland. The British Society of Gastroenterology (BSG) updated guidelines on the treatment and management of Primary Biliary Cholangitis (PBC) in 2018. ${ }^{1}$ We audited our PBC patients to assess management of patients with PBC, and identify areas for improvement.

Methods Patients were identified by searching PAS and the Northern Ireland Electronic Care Record (NIECR). ICD-10 code K74.3 was used - 'Chronic nonsupparative destructive cholangitis' - as primary and secondary diagnosis. We audited the notes of all patients who had a diagnosis of PBC in our hospital catchment area within the last 10 years based on recommendations from the 2018 BSG PBC guidelines.

Results A total of 51 patients with PBC were identified. Mean age 66 [age range 31 to 92], 11 male and 40 female [78\% F; $22 \% \mathrm{M}]$.

Of our patients, $84 \%$ were on Ursodeoxycholic Acid (UDCA), but only 54\% were on the correct dose of $13-15 \mathrm{mg} /$ $\mathrm{kg} /$ day of UCDA.

6 patients considered high risk on their LFT results has not been referred for second line therapy. 3 UDCA intolerant patients had not been referred for second line therapy.

98\% (50/51) had had baseline TFTs checked; 51\% (26/51) had had coeliac serology checked. Bone densitometry (DEXA scan) had been performed on $63 \%$ of our patients with PBC.

14 patients reviewed during our audit had passed away over the last 10 years. Of these 50\% (7/14) had died of PBC or a liver related illness.

Conclusions The majority of patients were on UDCA, but not all were on the correct dose. Of concern were the high risk patients and UDCA-intolerant patients who had not been referred onwards for consideration of second line therapy and consideration of liver transplant. COVID19 lockdown certainly negatively influenced the optimal management of PBC patient over the last 12 months. To improve our care, we have instituted a PBC proforma, to be used during clinics and aim to streamline all $\mathrm{PBC}$ patients into one specialist clinic, rather than the current multi-clinic, multi-consultant set up. The recent addition of a Fibroscan service will also benefit patient management.

\section{REFERENCE}

1. Hirschfield GM, Dyson JK, Alexander GJM et al. 2018, Gut, e-publication, 2018, page $0-27$

\section{PTH-53 EVALUATING THE EFFECT OF COVID ON AN UPPER GI TWO WEEK WAIT PATHWAY}

Amy Woods*, Sabina Beg, Anum Javed. Imperial College Healthcare Nhs Trust, London, UK

10.1136/gutjnl-2021-BSG.368

Introduction The covid-19 pandemic has necessitated a change in how healthcare provision is delivered. As such 